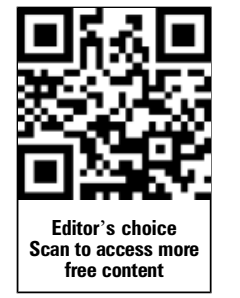

${ }^{1}$ Department of Medicine and Aging Science, Ophthalmic Clinic. University $\mathrm{G}$ d'Annunzio of Chieti-Pescara, Chieti, Italy ${ }^{2}$ Department of Health Science, University of Molise, Campobasso, Italy ${ }^{3}$ Ophthalmology Unit, Department of Neurological, Neuropsychological, Morphological and Movement Sciences, University of Verona, Verona, Italy

${ }^{4}$ Department of

Ophthalmology, University of Siena, Siena, Italy

\section{Correspondence to}

Dr Luca Agnifili, Department of Medicine and Aging Science, Ophthalmic Clinic. University $\mathrm{G}$ d'Annunzio of ChietiPescara, Via dei Frentani, 114, Chieti 66100, Italy; l.agnifili@unich.it

LA and VF equally contributed to this work.

Received 7 September 2012 Revised 19 October 2012 Accepted 23 November 2012 Published Online First 25 December 2012

\title{
In vivo confocal microscopy of meibomian glands in glaucoma
}

\author{
Luca Agnifili, ${ }^{1}$ Vincenzo Fasanella, ${ }^{1}$ Ciro Costagliola, ${ }^{2}$ Cristina Ciabattoni, ${ }^{1}$ \\ Rodolfo Mastropasqua, ${ }^{3}$ Paolo Frezzotti, ${ }^{4}$ Leonardo Mastropasqua ${ }^{1}$
}

\section{ABSTRACT}

Aim To investigate, using laser scanning confocal microscopy (LSCM), the morphological changes of meibomian glands (MGs) in patients with glaucoma.

Methods A total of 80 patients who were glaucomatous were enrolled, and 20 healthy subjects were used as controls. After completing an Ocular Surface Disease Index (OSDI) questionnaire, all subjects underwent tear film break-up time (BUT), fluorescein staining, Schirmer test I (STI) and LSCM examination of the MGs. The main outcome measures were: eyelid margin epithelial cell density, mean acinar density (MAD) and area (MAA), glandular orifice area, secretion reflectivity and inhomogeneous appearance of interstice and acinar wall. Results According to the number of anti-glaucoma medications they were taking, patients were divided into three groups: group 1 (30 eyes), one drug; group 2 (23 eyes), two drugs; group 3 (27 eyes), three or more drugs. LSCM showed lower MAD and MAA, greater secretion reflectivity and glandular orifice area in groups 2 and 3 than in controls $(p<0.05)$. The inhomogeneity of the interstice and acinar wall was significantly greater in all groups compared to controls $(p<0.05)$. Preserved prostaglandin analogues (PGAs) induced more pronounced modifications of all parameters than preservative free (PF)-PGAs $(p<0.05)$. No significant differences were found between preserved and PF- $\beta$-blockers. Significant relations were found among MAD, MAA, secretion reflectivity and OSDI score, BUT and ST $(p<0.05)$ and between secretion reflectivity and orifice area $(p<0.001)$.

Conclusions In vivo LSCM is an effective tool in revealing morphological changes of MGs induced by anti-glaucoma medications. Given the key role in the ocular surface health, the evaluation of MG status in patients who are glaucomatous is worthwhile.

\section{INTRODUCTION}

Meibomian glands (MGs) are holocrine glands embedded in the tarsal plate of the eyelids. These glands synthesise meibum, a lipoid secrete that forms the superficial layer of the tear film, preventing its excessive evaporation and functioning as a lubricant for the eyelids during blinking. MG dysfunction is one of the most diffuse causes of dry eye. ${ }^{1}$

In glaucoma, long-term treatment induces modifications of ocular surface tissues and adnexa, such as the conjunctiva, cornea, eyelids, periocular skin and MGs. $^{2-5}$ To date, the impact of anti-glaucoma medications on MGs has been investigated using clinical methodologies such as meibometry and non-contact meibography. ${ }^{6-8}$ These studies reported that antiglaucoma drugs induced morphological changes and dysfunction of MGs, leading to dry eye.
In vivo laser scanning confocal microscopy (LSCM) can non-invasively characterise structural tissue changes in many ocular surface diseases. ${ }^{9}$ In glaucoma, LSCM is used to analyse ocular surface alterations induced by treatment, filtering bleb functionality ${ }^{4} 10$ and trans-scleral aqueous outflow modifications induced by medical and surgical approaches. $^{11-14}$

Recently, LSCM was used to investigate MGs in contact lens (CL) wearers, in Sjögren syndrome and in MG dysfunction. ${ }^{15-18}$ However, to date, no previous study has evaluated MGs in glaucoma and the effects of topical medications by using in vivo confocal microscopy. The aim of this study was therefore to describe the features of MGs in medically controlled glaucoma by means of LSCM, in order to elucidate modifications induced by treatment.

\section{MATERIALS AND METHODS \\ Patient enrolment}

This was a case-control study. The study adhered to the tenets of the Declaration of Helsinki, and our institutional review board (Department of Medicine and Ageing Science, G d'Annunzio University of Chieti-Pescara, Chieti, Italy) approved the project. Written informed consent was obtained from all patients prior to enrolment. We examined 80 consecutive Caucasian patients (80 eyes) with medically controlled primary open angle glaucoma referred to our clinic; 20 healthy Caucasian subjects (20 eyes) were used as controls.

For patients who were glaucomatous, inclusion criteria were: corrected visual acuity $\geq 8 / 10$, refractive error $\leq 3$ dioptres, mean intraocular pressure (IOP) at the time of diagnosis ranging from 22-34 mm Hg and medically controlled at enrolment $(\mathrm{IOP}<18 \mathrm{~mm} \mathrm{Hg}$ : mean of three measurements taken at 9:00, 12:00 and 16:00), central corneal thickness (CCT) (ultrasound pachimetry: Altair; Optikon 2000, Rome, Italy) ranging from $530-570 \mu \mathrm{m}$, visual field (VF) test ((Humphrey field analyser II 750 (Carl Zeiss Meditec Inc, Dublin, California, USA) (30-2 test, full threshold)) showing at least three contiguous points on the total deviation probability plot at the less than $2 \%$ level, glaucoma Hemifield test 'outside normal limits' and classic ophthalmoscopic signs of glaucomatous optic consistent with the VF alterations. Topical treatment had to be the same in both eyes without variation during the 18 months immediately prior to enrolment.

Exclusion criteria were history of ocular or systemic diseases or treatments in the last 12 months that could have modified the MG status, previous ocular surgery and ocular trauma, end-stage 
glaucoma, pregnancy and CL wear. History of MG dysfunction (according to criteria reported by Matsumoto et al), ${ }^{17}$ and dry eye prior to glaucoma diagnosis and initiating treatment were also considered exclusion criteria.

Controls showed a best-corrected visual acuity $\geq 8 / 10$, a refractive error $\leq 3$ dioptres, mean IOP lower than $18 \mathrm{~mm} \mathrm{Hg}$, CCT ranging from 530-570 $\mu \mathrm{m}$, absence of signs of glaucomatous optic neuropathy and a normal VF examination. None of the healthy subjects had either a history of topical or systemic treatment nor were they affected with any ocular or systemic diseases in the last 12 months. Pregnant women and CL wearers were also excluded.

In patients who were glaucomatous and healthy controls, both eyes were evaluated, but only one eye per subject was randomly chosen (using a computer generated random number list) for the statistical analysis.

\section{Ocular Surface Disease Index (OSDI) questionnaire and tear film function tests}

After enrolment, all subjects were asked to complete the OSDI questionnaire. ${ }^{19}$ Subsequently, tear film break-up time (BUT) tests and the Schirmer test I (STI) with topical anaesthesia (20 min after BUT measurements) were performed. BUT was recorded as the average of three consecutive measurements. The STI result was expressed as the length of the strip that was wet after $5 \mathrm{~min}$; corneal staining was scored as previously described. ${ }^{20}$

\section{Slit lamp evaluation}

Using a slit lamp the MG orifice obstruction was evaluated by applying digital pressure on the lower tarsum. ${ }^{15}{ }^{16}$ The degree of ease in expressing meibomian secretion (meibum score or MG expressibility) was evaluated semiquantitatively as follows: grade 0, clear meibum easily expressed; grade 1, cloudy meibum expressed with mild pressure; grade 2 , cloudy meibum expressed with more than moderate pressure; and grade 3, meibum not expressed even with hard pressure.

\section{Meibography}

Transillumination of the MGs through the inferior lid was achieved by using an illuminated probe placed on the skin of the everted eyelid. The glandular morphology was observed and photographed along the entire length of the eyelid using a slit lamp. This allowed quantification of the meibomian glandular rows and their loss.

The degree of MG dropout (meibo score) was evaluated as follows: grade 0 , no dropout; grade 1 , dropout in less than half of the inferior tarsum; and grade 2, dropout in more than half of the inferior tarsum. ${ }^{15}$ The meibo score for the lower eyelid was summed to obtain a score for each eye.

\section{In vivo confocal microscopy}

The day after the tear film function assessment, LSCM was performed using HRT III Rostock Cornea Module (Heidelberg Engineering GmbH, Dossenheim, Germany).

Briefly, after the lower eyelid was partly everted, the centre of the sterile Tomo-Cap was applanated onto the centre of the eyelid margin. The instrument was manually focused while the microscope was in 'section mode' of acquisition modality.

Scanning was started at the most superficial tissues and progressed down to the deepest tissue that could be visualised with a satisfactory resolution. The procedure was repeated by moving the lens along the entire lid length (from nasal to temporal canthus) with minute horizontal movements. A total of 15 images (5 each for the middle, nasal and temporal areas) were acquired per lid at every $10 \mu \mathrm{m}$ of depth for a maximum depth of $30 \mu \mathrm{m}$. Images were also acquired in the mid-depth range in order to manually assess the quality of the different structures found in this area. Images were $384 \times 384$ pixels with a $400 \times 400 \mu \mathrm{m}$ field of view. Typical sessions for LSCM examination lasted between 3-5 min. A single operator (VF) performed confocal examinations and selected the images, which were evaluated by a second operator (LA). Both operators were masked for patient history and to the group.

Three randomly selected, non-overlapping high-quality images of the nasal, middle and temporal lower eyelid margins were analysed. LSCM parameters considered were: (1) cellular density of the superficial and basal epithelium of the eyelid margin (calculated automatically at the largest available region (Cell Count software; Heidelberg Engineering)); (2) the mean acinar area of MG (MAA) (area of each acinar unit calculated using ImageJ, an open source software program (http://rsb.info. nih.gov/ij/), as described elsewhere); ${ }^{21}$ (3) mean acinar density (MAD) (density of the acinar unit manually marked for each $400 \times 400 \mu \mathrm{m}$ frame and calculated automatically using Cell Count); (4) area of glandular orifice (calculated by using ImageJ); (5) meibum secretion reflectivity; (6) inhomogeneity of periglandular interstices and (7) acinar unit wall. An arbitrary grading scale of 1-4 was adopted to quantify secretion reflectivity and inhomogeneity. ${ }^{15} 16$

To validate the sectorial findings, we manually created a planar reconstruction of the central part of the lower eyelid (3 $\mathrm{mm}$ in length) in two healthy and four glaucomatous eyes (two under monotherapy and two under multitherapy).

\section{Statistical analysis}

Analysis was performed using SPSS V.13.0 Software (SPSS Inc.; Chicago, Illinois, USA). Student $t$ and $\chi^{2}$ tests were used to evaluate age and sex differences, respectively, among healthy and patients who were glaucomatous.

The Mann-Whitney $U$ test was used to determine differences among groups of subjects. Spearman's correlation analysis was used to investigate the relations between LSCM parameters and OSDI score, BUT, ST and corneal staining and among LSCM parameters. $p$ Values less than 0.05 were considered statistically significant.

Multivariate regression analysis was performed with stepwise selection of contributing variables (probability of $\mathrm{F}$ to enter $\leq 0.050$, probability of $F$ to remove $\geq 0.100$ ) including age, LSCM parameters, OSDI score, BUT, STI value and the duration of topical treatment.

\section{RESULTS}

The demographic and clinical data in glaucoma groups are shown in table 1. Patient treatment is shown in table 2. No

Table 1 Demographics and clinical data of healthy controls and glaucoma groups

\begin{tabular}{llllll}
\hline & $\begin{array}{l}\text { Age, } \\
\text { years } \pm S D\end{array}$ & $\begin{array}{l}\text { Gender, } \\
\text { M/F }\end{array}$ & $\begin{array}{l}\text { IOP, } \mathbf{m m} \\
\mathrm{Hg} \pm \text { SD }\end{array}$ & $\begin{array}{l}\text { MD, } \\
\mathrm{dB} \pm \text { SD }\end{array}$ & $\begin{array}{l}\text { Mean } \\
\text { treatment time, } \\
\text { months } \pm S D\end{array}$ \\
\hline Controls & $61.1 \pm 9.4$ & $10 / 10$ & $14.9 \pm 3.0$ & $1.03 \pm 0.34^{*}$ & $\mathrm{NA}$ \\
Group 1 & $60.6 \pm 9.1$ & $14 / 16$ & $13.9 \pm 3.1$ & $-2.84 \pm 1.12$ & $52.7 \pm 9.43$ \\
Group 2 & $59.9 \pm 9.3$ & $11 / 12$ & $15.1 \pm 1.9$ & $-3.04 \pm 0.65$ & $50.1 \pm 10.4$ \\
Group 3 & $62.6 \pm 10.0$ & $14 / 13$ & $15.4 \pm 4.4$ & $-5.12 \pm 0.89$ & $51.2 \pm 12.7$ \\
\hline
\end{tabular}

${ }^{*} \mathrm{p}<0.05$ vs groups 1,2 and 3 .

$\mathrm{dB}$, decibel; IOP, intraocular pressure; $\mathrm{MD}$, mean defect; $\mathrm{NA}$, not applicable. 
Table 2 Patient treatment

\begin{tabular}{|c|c|c|}
\hline Group & Treatment & $\mathbf{N}$ \\
\hline \multirow[t]{7}{*}{ Group 1} & Single & 30 \\
\hline & $\beta$-blockers & 15 \\
\hline & Preserved timolol $0.5 \%$ & 8 \\
\hline & PF-timolol $0.5 \%$ & 7 \\
\hline & PGAs & 13 \\
\hline & Latanoprost $0.005 \%$ & 8 \\
\hline & PF-tafluprost $0.0015 \%$ & 7 \\
\hline \multirow[t]{5}{*}{ Group 2} & Double & 23 \\
\hline & Latanoprost-timolol fixed combination & 7 \\
\hline & Bimatoprost and timolol unfixed combination & 7 \\
\hline & Brimonidine and timolol unfixed combination & 4 \\
\hline & Dorzolamide-timolol fixed combination & 5 \\
\hline \multirow[t]{4}{*}{ Group 3} & Triple or more & 27 \\
\hline & Bimatoprost $0.03 \%$, brimonidine and timolol $0.05 \%$ & 14 \\
\hline & Latanoprost $0.005 \%$, timolol and dorzolamide & 7 \\
\hline & Bimatoprost $0.03 \%$, brimonidine, timolol $0.05 \%$ and dorzolamide & 6 \\
\hline
\end{tabular}

patients in group 1 had their treatment modified from treatment onset.

OSDI questionnaire score, STI and BUT were significantly different among glaucoma groups and controls and between group 1 with groups 2 and $3(\mathrm{p}<0.05)$. Corneal staining and meibum and meibo scores in the glaucoma groups were significantly different compared to controls, but were not different among groups (table 3).

\section{LSCM of eyelid epithelium}

Epithelial cellular densities were significantly lower in groups 2 and 3 compared to controls $(\mathrm{p}<0.05)$ and in group 2 compared to group 1. No significant differences were found between controls and group 1 and between groups 2 and 3 (table 4); (figure 1). No significant differences were found among drug classes in group 1 (table 5).

\section{LSCM of MGs}

MAD and MAA were significantly lower in groups 2 and 3 than in controls $(p<0.05)$. Significantly lower values were found between all preserved drugs of group 1 and controls and between preserved prostaglandin analogues (PGAs) and controls $(p<0.05)$ (tables 4 and 5); (figure 2). In group 1, MAD and MAA were lower in preserved PGAs than in preservative free
(PF)-PGAs $(\mathrm{p}<0.05)$ (table 5). Secretion reflectivity was significantly greater in groups 2 and 3 than in group 1 and controls $(p<0.001)$. In group 1 , patients treated with preserved PGAs showed higher reflectivity with respect to controls $(p<0.05)$ (figure 2). No statistically significant differences were found between preserved and PF- $\beta$-blockers.

The glandular orifice area appeared significantly greater in groups 2 and 3 with respect to controls and group $1(p<0.05)$. No significant differences were found among drugs in group 1 (figure 1). MAD, MAA, reflectivity and orifice area were not significantly different between groups 2 and 3 and between overall group 1 and controls.

The inhomogeneity of interstice and MG wall was significantly higher in all groups than in controls, with values higher in group 3 compared to groups 2 and $1(\mathrm{p}<0.05)$. In group 1 , overall preserved monotherapy induced higher inhomogeneity compared to PF formulations; only preserved PGAs showed values significantly higher compared to controls $(p<0.05)$. Preserved PGAs showed higher inhomogeneity than PF-PGAs $(p<0.05)$, whereas no significant differences were found between preserved and PF- $\beta$-blockers (figure 3 ).

The global distribution of MGs (central part of the inferior eyelid margin) in healthy subjects and modifications induced by monotherapy and multitherapy in patients who were glaucomatous are shown in figure 4 (figure $4 \mathrm{~A}-\mathrm{C}$, respectively).

The OSDI score, STI and BUT correlated with MAD, MAA and secretion reflectivity $(\mathrm{p}<0.05$, Spearman). The inhomogeneity of the interstice correlated with secretion reflectivity and BUT $(p<0.05$, Spearman); secretion reflectivity and glandular orifice area were strongly correlated $(p<0.001)$. Multivariate regression analysis indicated that LSCM parameters did not significantly correlate with age in neither healthy nor glaucoma groups $(\mathrm{p}>0.05)$.

\section{DISCUSSION}

Recent studies have shown that anti-glaucoma medications induce MG loss and low secretion expressibility. ${ }^{6-8}$ These findings correlated with BUT and ocular surface symptoms. Notably, the glandular dropout was not significantly different between preserved PGAs and preserved $\beta$-blockers. ${ }^{7} 8$ However, these studies did not consider the eyes of patients taking PF drugs and, therefore, the question of whether the gland modifications were induced by preservative compounds, active compounds or by both was not clarified. ${ }^{8}$ Unexpectedly, MG modifications did not correlate with the number of daily drugs since the meibum and meibo scores did not differ among treatment groups.?

Our study, which analysed MGs in glaucoma using in vivo confocal microscopy for the first time, showed an overall

Table 3 Clinical characteristics

\begin{tabular}{lcccccc}
\hline & OSDI score & BUT & STI & Corneal staining & Meibo score & Meibum score \\
\hline Controls & $9.5 \pm 4.8^{*}$ & $12.0 \pm 2.1^{*}$ & $18.0 \pm 5.0^{*}$ & $0.3 \pm 1.1^{*}$ & $0.12 \pm 0.03^{*}$ & $1.30 \pm 0.72^{*}$ \\
Group 1 & $16.4 \pm 5.4^{* *}$ & $7.2 \pm 1.9^{* *}$ & $9.3 \pm 5.1^{* *}$ & $1.9 \pm 2.3$ & $0.48 \pm 0.32$ & $2.05 \pm 0.91$ \\
PF drugs & $9.4 \pm 3.5^{* * *}$ & $7.7 \pm 1.8^{* * * *}$ & $9.1 \pm 3.1^{* * * *}$ & $1.9 \pm 2.2$ & $0.44 \pm 0.41$ & $1.98 \pm 0.68$ \\
Preserved drugs & $18.7 \pm 4.1^{* * *}$ & $6.1 \pm 1.9^{* * * *}$ & $9.4 \pm 6.1^{* * * *}$ & $1.9 \pm 1.8$ & $0.53 \pm 0.44$ & $2.12 \pm 1.03$ \\
Group 2 & $30.8 \pm 6.7$ & $4.1 \pm 1.5$ & $7.2 \pm 2.7$ & $2.3 \pm 2.1$ & $0.54 \pm 0.38$ \\
Group 3 & $32.6 \pm 7.2$ & $3.9 \pm 1.5$ & $6.8 \pm 2.7$ & $2.2 \pm 1.9$ & $0.57 \pm 0.63$ \\
\hline
\end{tabular}

${ }^{*} p<0.05$ vs groups 1,2 and 3 .

$* * p<0.05$ vs groups 2 and 3 .

$* * * p<0.05$ vs group 1 preserved drugs and vs groups 2 and 3 .

$* * * * p<0.05$ vs groups 2 and 3 .

BUT, break-up time; OSDI, Ocular Surface Disease Index; PF, preservative free; STI, Schirmer test I. 


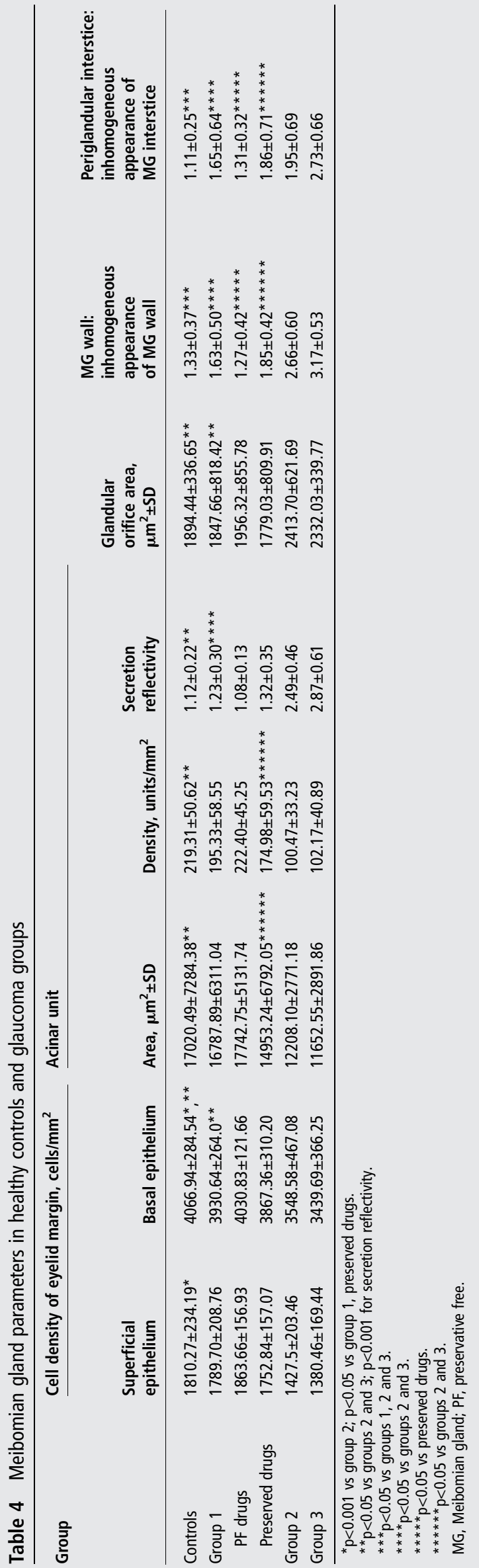

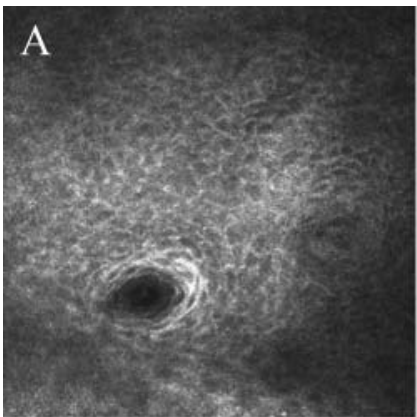
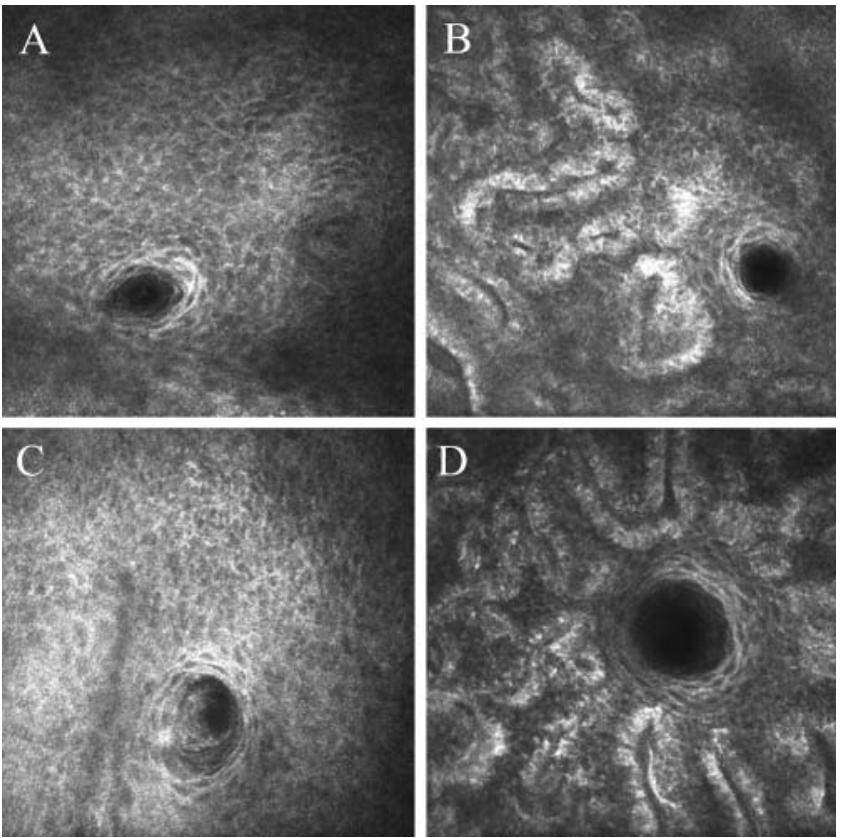

Figure 1 Epithelium of basal eyelid margin and glandular orifice. The basal epithelial cell density appeared reduced and glandular orifice area greater in groups 2 and 3. (A) Controls; (B-D) groups 1 to 3, respectively. In glaucoma groups epithelial cells presented with smaller size, polygonal shapes, and less distinguished and hyper-reflective borders.

reduction of the MAD and MAA, particularly in patients in multitherapy. In contrast, overall group 1 did not differ compared to controls.

Lower MAD and MAA values are expressions of glandular loss and reduced meibum production, respectively. This could explain the higher secretion reflectivity in groups 2 and 3, which indicated increased secretion viscosity. Consequently, the higher glandular orifice area in groups 2 and 3 was probably an adaptive mechanism to overcome the high secretion density and the duct blockage induced by treatment.

These findings were supported by the correlation between the secretion reflectivity and glandular orifice area. These results have an evident clinical impact since MAD, MAA and reflectivity correlated with OSDI, BUT and ST.

All groups showed an inhomogeneous appearance of the interstice and glandular wall compared to controls. As suggested by Villani et $a l^{15} 16$ for others conditions, the inhomogeneity may be seen as a sign of tarsum and MG inflammation.

Interestingly, these were the only parameters that also differed between group 1 and controls and between groups 2 and 3. This appears consistent with the dose-dependent inflammatory effects of anti-glaucoma medications on ocular surface. ${ }^{2} 22$ Nevertheless, in our cases, it was difficult to identify inflammatory cells with the typical dendritic shape.

Important differences arose concerning preserved and PF medications in group 1: though overall the monotherapy did not alter MAD, MAA and secretion reflectivity, the drug class analysis showed that preserved drugs were more toxic than $\mathrm{PF}$ formulations, with preserved PGAs being more toxic than preserved $\beta$-blockers. Conversely, no differences were found between PF-PGA and PF- $\beta$-blockers.

The comparison of preserved and PF agents of the same drug class showed that preserved PGAs were more toxic than PF-PGAs, without significant differences between preserved and 


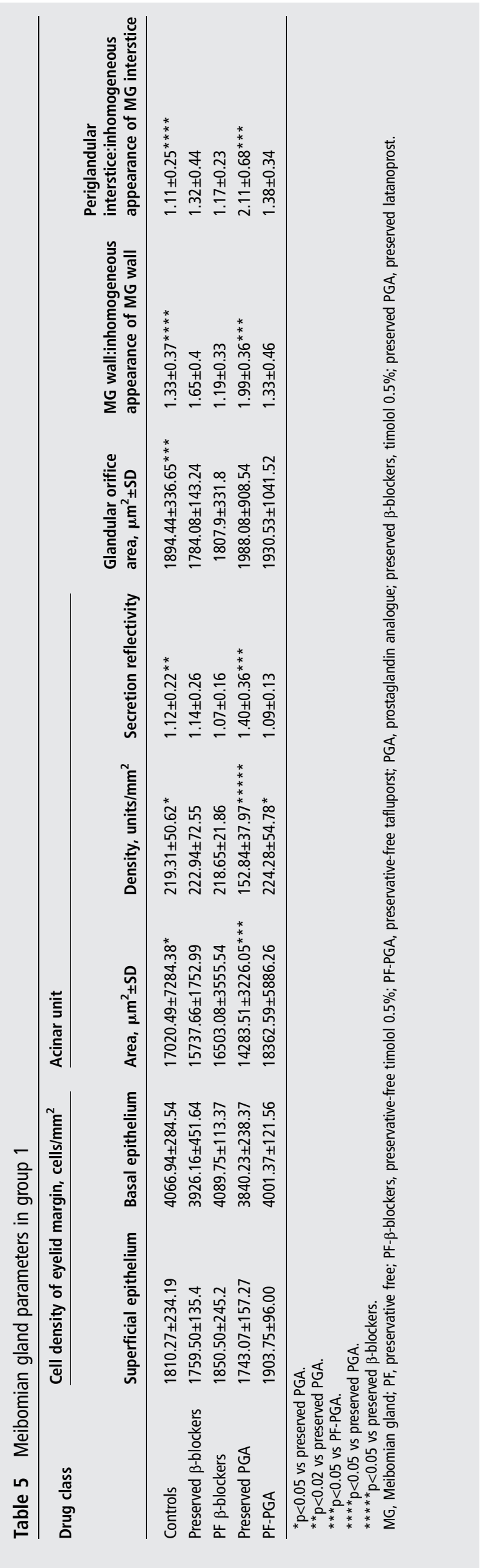

PF- $\beta$-blockers. These aspects were not consistent with meibography in our study and Arita et al's findings, which reported similar MG dropout in preserved PGA-treated and $\beta$-blocker-treated eyes. ${ }^{6-8}$ One may hypothesise that LSCM provided a more accurate definition of MGs than clinical approaches. However, one cannot exclude the influence of treatment duration, since the duration was significantly shorter in our study.

Concerning markers of inflammation, we observed that overall preserved drugs induced higher inhomogeneity compared to overall PF formulations, which, conversely, did not differ from controls. When comparing drug classes, preserved PGAs were more inflammatory than PF-PGAs, without significant differences between preserved and PF- $\beta$-blockers.

We propose that PGAs play a key role in inducing MG alterations. Nonetheless, one cannot exclude a primary and dosedependent effect of benzalkonium chloride (BAK), which is more concentrated in preserved PGAs than in $\beta$-blockers, though the latter are administered twice daily. A hypothetical synthesis could be that BAK either exerts a direct toxic effect on MGs or promotes the drug's penetration into the glands, allowing PGAs to induce final damage.

The mechanisms leading to MG alterations remain unclear, though drug-induced inflammation, ${ }^{23} 24$ allergy and toxicity within conjunctiva ${ }^{25}{ }^{26}$ might diffuse for contiguity to tarsum and acinar units. As supposed, such processes may cause meibum stagnation followed by the keratinisation of $\mathrm{MG}$ orifices. $^{27} 28$

Consistently, our results indicated that inflammation might be the first step in a cascade of glandular modifications, since inhomogeneity was the only marker that differentiated group 1 from controls. However, one cannot exclude that MG changes were secondary to allergy or inflammation, instead of a direct effect of medications.

A noteworthy consideration was that MG loss, high secretion reflectivity and orifice area, and high inhomogeneity of the glandular wall and interstice, were also observed in MG dysfunction. $^{15} 17$

Thus, MG dysfunction and the effects of anti-glaucoma treatment on MGs differ only by the acinar unit size. The same findings were reported also in CL wearers, ${ }^{16}$ where CL use induced a mechanical obstructive MG disease, though the acinar diameter decreased.

We hypothesise that the reduced acinar unit area in patients with glaucoma may be a drug-induced glandular activity loss, with decreased meibum production and consequent secretion hyperviscosity.

Differently, in MG dysfunction the gland productivity continues, but meibum cannot be secreted. This leads to the acinar unit dilation. However, we cannot rule out that in the very early periods after initiating treatment, MGs may also increase the acinar unit area in patients with glaucoma.

Hence, anti-glaucoma treatment seems to induce an incomplete obstructive-like MG condition, which resulted in evaporative dry eye. This aspect appeared to be supported by the correlations among confocal parameters and clinical data.

Our study presents some limitations. First, this is a crosssectional study with a similar longevity of disease in all groups: thus, we cannot analyse the effects of length of treatment. Second, the MG status before the start of treatment was not known. Third, we did not include control groups receiving vehicles at different BAK concentrations, which would have been useful in the definition of the exact role of preservative and active compounds. 

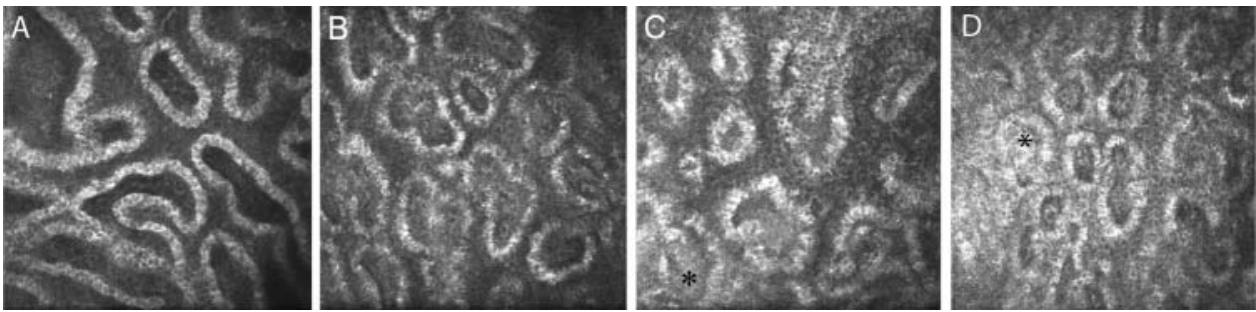

Figure 2 Mean acinar density, mean acinar area and secretion reflectivity. Acinar unit density and area appeared reduced and meibomian gland secretion (asterisks) was hyper-reflective in groups 2 and 3 ((A) controls; (B-D) groups 1 to 3, respectively) compared to controls.

Figure 3 Inhomogeneity of meibomian gland (MG) wall and periglandular interstice. MG wall and periglandular interstice presented an inhomogeneous appearance, which progressively increased with the number of drugs used for treatment. (A) Controls; (B-D) groups 1 to 3, respectively.
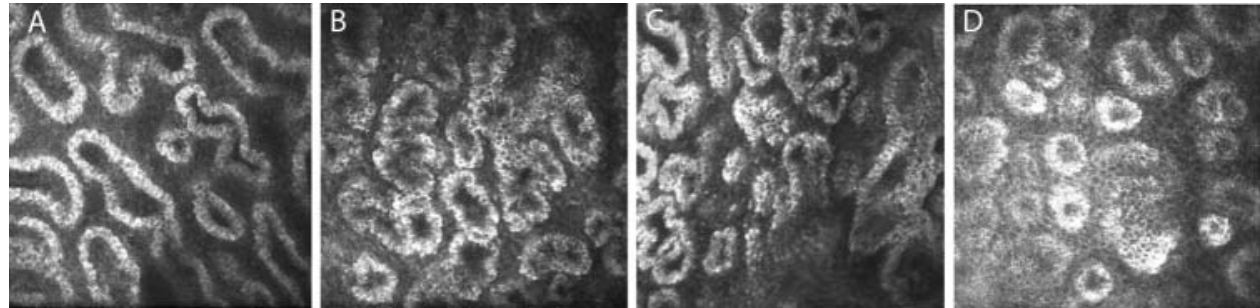

Finally, we cannot clearly identify the initial site of damage and the first MG modification, though the high inhomogeneity also seen in group 1 indicated that the interstice and acinar unit wall might be altered early. Further prospective studies evaluating the initial MG status in treatment-naïve eyes and the impact of medications over time are mandatory to clarify these points.
In conclusion, LSCM proved valuable in identifying MG modifications induced by anti-glaucoma drugs, possibly more accurate than just a clinical approach. These modifications present evident implications for the ocular surface health; therefore, where available, the use of PF formulations is advisable. Further studies evaluating the relation between MG changes and
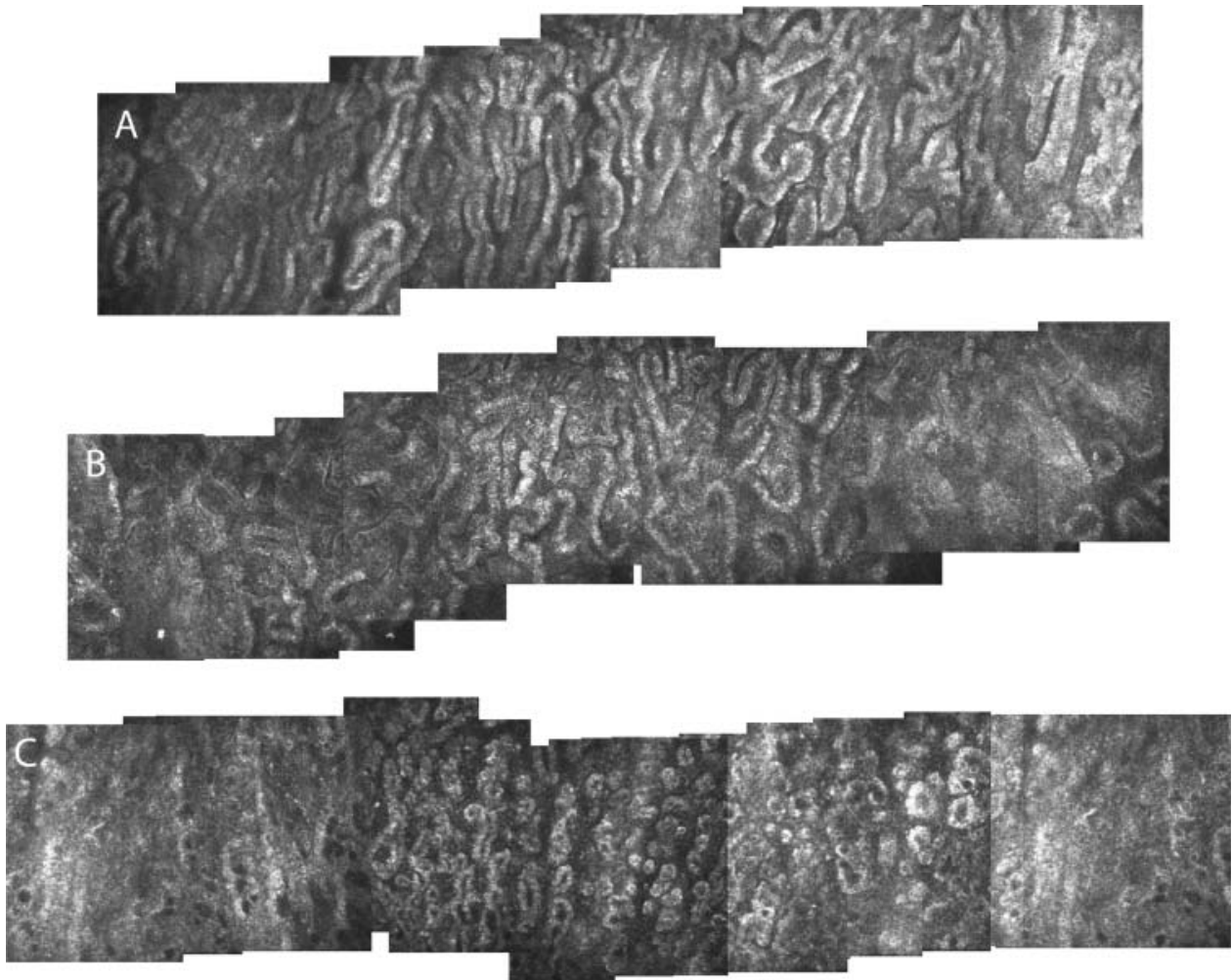

Figure 4 Planar reconstruction of meibomian glands (MGs). The reconstruction shows the global distribution of MGs within the central part of the inferior eyelid margin. The final map was obtained by juxtaposing acquired images, consecutively moving the cornea module from the centre to the nasal and temporal region (right and left side of the image, respectively) and from the lid margin to the fornix (bottom and top of the image, respectively). Topical medications diffusely affected MG morphology, reducing density and area, increasing secretion reflectivity and promoting a marked glandular and periglandular status of inflammation. (A) Controls; (B) 48 months of treatment with one drug; (C) 52 months of treatment with three drugs. 
adherence to and persistence of treatment could clarify the role of MG damage on worsening patient compliance.

Contributors LA: conception and design; writing the article; critical revision of the article; VF: provision of patients; performing examinations; CCo: writing the article; final approval of the article; CCi: provision of patients; data collection; RM: literature research; data collection; PF: literature research, critical revision of the article; LM: conception and design, final approval of the article.

\section{Funding None.}

Competing interests None.

Patient consent Obtained.

Ethics approval The study adhered to the tenets of the Declaration of Helsinki and our institutional review board (Department of Medicine and Ageing Science, G d'Annunzio University of Chieti-Pescara, Chieti, Italy) approved the project.

Provenance and peer review Not commissioned; externally peer reviewed.

\section{REFERENCES}

1 McCulley JP, Shine WE. Meibomian gland function and the tear lipid layer. Ocul Surf 2003;1:97-106.

2 Baudouin C, Labbé A, Liang $\mathrm{H}$, et al. Preservatives in eyedrops: the good, the bad and the ugly. Prog Retin Eye Res 2010;29:312-34.

3 Herreras JM, Pastor JC, Calonge M, et al. Ocular surface alteration after long-term treatment with an antiglaucomatous drug. Ophthalmology 1992;99:1082-8.

4 Ciancaglini M, Carpineto P, Agnifili L, et al. An in vivo confocal microscopy and impression cytology analysis of preserved and unpreserved levobunolol-induced conjunctival changes. Eur J Ophthalmol 2008;18:400-7.

5 Alm A, Grierson I, Shields MB. Side effects associated with prostaglandin analog therapy. Surv Ophthalmol 2008;53(Suppl 1):S93-105.

6 Cunniffe MG, Medel-Jiménez R, González-Candial M. Topical antiglaucoma treatment with prostaglandin analogues may precipitate meibomian gland disease. Ophthal Plast Reconstr Surg 2011;27:e128-9.

7 Arita R, Itoh K, Maeda S, et al. Comparison of the long-term effects of various topical antiglaucoma medications on meibomian glands. Cornea 2012;31:1229-34.

8 Arita R, Itoh K, Maeda S, et al. Effects of long-term topical anti-glaucoma medications on meibomian glands. Graefes Arch Clin Exp Ophthalmol 2012;250:1181-5

9 Kaufman SC, Musch DC, Belin MW, et al. Ophthalmic technology assessment committee cornea panel. Confocal microscopy: a report by the American Academy of Ophthalmology. Ophthalmology 2004;111:396-406.

10 Ciancaglini M, Carpineto P, Agnifili L, et al. Filtering bleb Functionality: a clinical, anterior segment optical coherence tomography and in vivo confocal microscopy study. J Glaucoma 2008;17:308-17.
11 Ciancaglini M, Carpineto P, Agnifili L, et al. Conjunctival modifications in ocular hypertension and primary open angle glaucoma: an in vivo confocal microscopy study. Invest Ophthalmol Vis Sci 2008;49:3042-8.

12 Ciancaglini M, Carpineto P, Agnifili L, et al. Conjunctival characteristics in primary open angle glaucoma and modifications induced by trabeculectomy with mitomycin C: an in vivo confocal microscopy study. Br J Ophthalmol 2009;93:1204-9.

13 Mastropasqua L, Agnifili L, Ciancaglini $\mathrm{M}$, et al. In vivo analysis of conjunctiva in gold micro shunt implantation for glaucoma. Br I Ophthalmol 2010;94:1592-6.

14 Mastropasqua L, Agnifili L, Salvetat ML, et al. In vivo analysis of conjunctiva in canaloplasty for glaucoma. Br I Ophthalmol 2012;96:634-9.

15 Villani E, Beretta S, De Capitani M, et al. In vivo confocal microscopy of meibomian glands in Sjögren's syndrome. Invest Ophthalmol Vis Sci 2011;52:933-9.

16 Villani E, Ceresara G, Beretta $S$, et al. In vivo confocal microscopy of meibomian glands in contact lens wearers. Invest Ophthalmol Vis Sci 2011;52:5215-19.

17 Matsumoto Y, Sato EA, Ibrahim OM, et al. The application of in vivo laser confocal microscopy to the diagnosis and evaluation of meibomian gland dysfunction. Mol Vis 2008;14:1263-71.

18 Ibrahim OM, Matsumoto Y, Dogru M, et al. The efficacy, sensitivity, and specificity of in vivo laser confocal microscopy in the diagnosis of meibomian gland dysfunction. Ophthalmology 2010;117:665-72.

19 Schiffman RM, Christianson MD, Jacobsen G, et al. Reliability and validity of the ocular surface disease index. Arch Ophthalmol 2000;118:615-21.

20 The definition and classification of dry eye disease: report of the definition and classification subcommittee of the international dry eye workshop (2007). Ocul Surf 2007:5:75-92.

21 Messmer EM, Zapp DM, Mackert MJ, et al. In vivo confocal microscopy of filtering blebs after trabeculectomy. Arch Ophthalmol 2006;124:1095-103.

22 Pisella PJ, Pouliquen P, Baudouin C. Prevalence of ocular symptoms and signs with preserved and preservative free glaucoma medication. $\mathrm{Br} J$ Ophthalmol 2002:86:418-23.

23 Sherwood MB, Grierson I, Millar L, et al. Long-term morphologic effects of antiglaucoma drugs on the conjunctiva and Tenon's capsule in glaucoma patients. Ophthalmology 1989;96:327-35.

24 Brandt JD, Wittpenn JR, Katz LJ, et al. Conjunctival impression cytology in patients with glaucoma using long-term topical medication. Am J Ophthalmol 1991;112:297-301.

25 Baudouin C, de Lunardo C. Short-term comparative study of topical $2 \%$ carteolol with and without benzalkonium chloride in healthy volunteers. Br J Ophthalmol 1998:82:39-42.

26 Broadway DC, Grierson I, O'Brien C, et al. Adverse effects of topical antiglaucoma medication. I. The conjunctival cell profile. Arch Ophthalmol 1994;112:1437-45.

27 Jester JV, Nicolaides N, Smith RV. Meibomian gland studies: histologic and ultrastructural investigations. Invest Ophthalmol Vis Sci 1981;20:537-47.

28 Nicolaides N, Santos EC, Smith RE, et al. Meibomian gland dysfunction, III. Meibomian gland lipids. Invest Ophthalmol Vis Sci 1989:30:946-51. 